\section{Foscarnet-induced genital lesions: An overview with a case report}

\author{
Jonas A. Adalsteinsson, Michael Pan, \\ Shivani Kaushik, Jonathan Ungar \\ Department of Dermatology, Mount \\ Sinai, New York City, NY, USA
}

\begin{abstract}
Foscarnet is an important antiviral medication used mainly in the treatment of complicated Herpes-simplex virus and cytomegalovirus (CMV) infections. Reported first in the 1990's, genital ulcers are a potential side effect in about $10 \%$ of cases. We report the case of a 29 year old man with acute myelogenous leukemia who was on ganciclovir for CMV prophylaxis. Three weeks after being switched to foscarnet because of neutropenia, he developed two, painful symmetric ulcers on the inferior aspect of glans penis. Viral and bacterial cultures were negative. Two weeks after stopping the infusion of foscarnet, the ulcers subsided without any additional treatment. It is important that physicians be aware of this potentially disfiguring side effect of foscarnet so that methods of prevention can be implemented early in the treatment of these patients.
\end{abstract}

\section{Introduction}

Foscarnet usage has fallen out of favor given the better safety profile of other antiviral medictions. In cases of acyclovir or ganciclovir resistance, foscarnet has an important role to play in the treatment or prophylaxis of Herpes-simplex virus (HSV) and cytomegalovirus (CMV) infections. Foscarnet not only inhibits proliferation of all herpes viruses, but also of human immunodeficiency virus (HIV), influenza viruses and the hepatitis B virus. It has low oral bioavailability and thus must be administered intravenously and is excreted in over $90-95 \%$ unchanged form through the kidneys. ${ }^{1,2}$ In addition to genital ulcers, frequently reported serious adverse effects are nephrotoxicity, electrolyte disturbances, nausea and seizures. ${ }^{2,3}$ Other side effects include arrhythmias or seizures that may be an effect of transient decreases in serum ionized calcium levels, which appear to occur in all patients during infusion of the drug. ${ }^{4}$ A less common complication of treatment seen in approximately $10 \%$ of cases is genital ulcerations. ${ }^{1,5}$ In 1990 , the first cases were reported in AIDS patients being treated with foscarnet for CMV retinitis. The patients developed $1-5 \mathrm{~cm}$ tender lesions of the glans that were negative in the workup for any infectious disease process. This side effect of foscarnet was uncovered when most lesions healed in 12-14 days after discontinuing the drug. ${ }^{6,7}$ Many similar cases were subsequently reported between 19901996, mostly in AIDS affected homosexual males. ${ }^{2,8-13}$

We report the case of a 29 year old man with acute myelogenous leukemia (AML) who developed penile ulcerations after being treated with foscarnet for $\mathrm{CMV}$ viremia.

Patient's consent was obtained before writing this case report.

\section{Case Report}

A 29 year old Hispanic man with AML complained of a sudden appearance of painful lesions on his penis. He was diagnosed with AML one year earlier and completed an allogenic stem cell transplatation one month prior to current admission. His posttransplant clinical course was complicated by neutropenic fever of unknown origin. He was treated empirically with vancomycin and aztreonam and prophylactically with acyclovir, posaconazole and TMP/SMX. Initial serum PCR studies for CMV DNA showed low grade viremia at $376 \mathrm{IU} / \mathrm{mL}$. Repeat PCR one week later showed CMV reactivation at $1522 \mathrm{IU} / \mathrm{mL}$. Acyclovir was discontinued and foscarnet was started at 90 $\mathrm{mg} / \mathrm{kg}$ every $12 \mathrm{~h}$ at this point to avoid bone marrow toxicity with ganciclovir. Patient was pre-hydrated using $500 \mathrm{cc}$ of normal saline with every foscarnet infusion. Repeat CMV PCR studies continued to show viremia.

On day 22 of foscarnet treatment, patient complained of painful lesions on his penis (Figure 1). Dermatology was consulted and physical examination revealed two symmetric erythematous shallow ulcers adjacent to the urethral meatus, oriented posteriorly toward the outward angle of the sub preputial space. The patient is uncircumcised. Viral culture was negative for HSV. Syphilis serology was negative. Foscarnet was continued despite appearance of these lesions due to persistent viremia for a total treatment duration of thirty days, after which the patient was switched back to acyclovir. The ulcers were monitored after stopping foscarnet. Nine days after cessation of foscarnet therapy the ulcers had notably improved (Figure 2).
Correspondence: Jonas A. Adalsteinsson, Department of Dermatology, Mount Sinai, 5E 98th St, New York City, NY 10029, USA. E-mail: jonas.adalsteinn@gmail.com

Key words: Foscarnet; ulcer; genital.

Conflicts of interest: the authors declare no potential conflicts of interest.

Received for publication: 20 May 2018 Accepted for publication: 4 June 2018.

This work is licensed under a Creative Commons Attribution-NonCommercial 4.0 International License (CC BY-NC 4.0).

(C) Copyright J.A. Adalsteinsson et al., 2018

Licensee PAGEPress, Italy

Dermatology Reports 2018; 10:7749

doi:10.4081/dr.2018.7749

After 16 days, the lesions had almost completely resolved without scarring (Figure 3). No topical therapy was administered for the ulcers.

\section{Discussion}

The reported incidence of foscarnetinduced genital lesions in males has been reported to be around $10 \%$. In one study, foscarnet was given to 132 patients with AIDS. 15 patients developed penile ulcerations with twelve of them doing so during the initial period of high dose treatment. All patients were uncircumcised. ${ }^{5}$ The main theory behind the pathophysiology of foscarnet-induced ulcers and erosions is that the drug itself induces an acute irritant contact dermatitis. Clinically the lesions can look similar to a fixed drug eruption but a biopsy will typically show a spongiotic dermatitis, rather than an interface dermatitis. Topical preperations of $3 \%$ foscarnet cream have been found to induce a severe irritant dermatitis when applied to skin and mucosal surfaces. This, in combination with the fact that approximately $90-95 \%$ of the drug is excreted unchanged in the urine ${ }^{2}$ contributes to the unique morphology of the lesions (Figure 1-3), which are often linearly distributed lesions outward from the urethral meatus. In uncircumcised men, urine that is eliminated through the urethral meatus has the potential to accumulate under the foreskin. It is in this population that most cases have been reported. Retention of the drug in the sub-preputial space after urination may account for the tendency of ulceration to occur on the glans. Our patient's hygiene was noted as poor, 


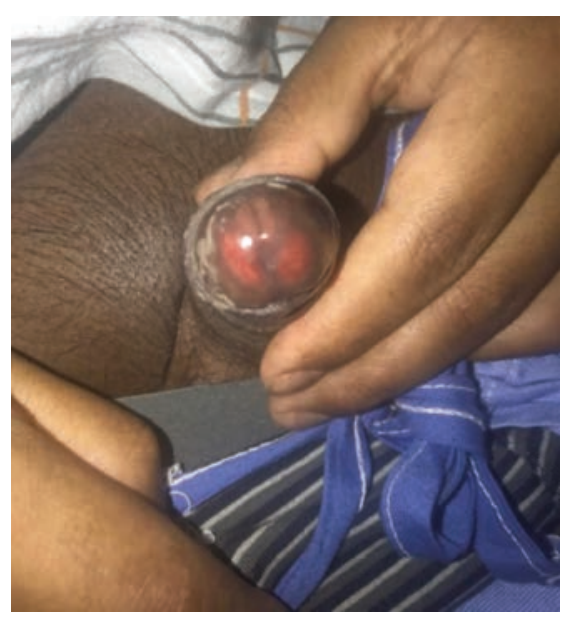

Figure 1. Day 22 of foscarnet therapy $(10 / 4)$. The patient had erythematous periurethral ulcers on his glans.

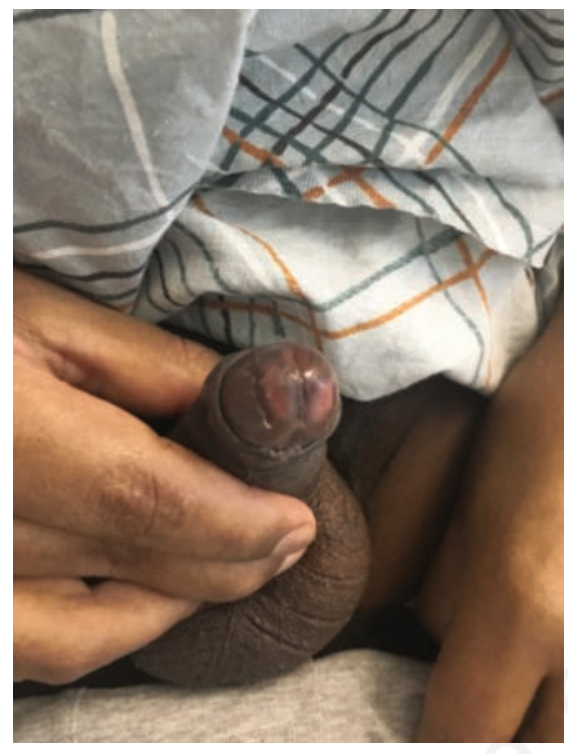

Figure 2. 9 days after stopping foscarnet the ulcers had partially healed (10/13).

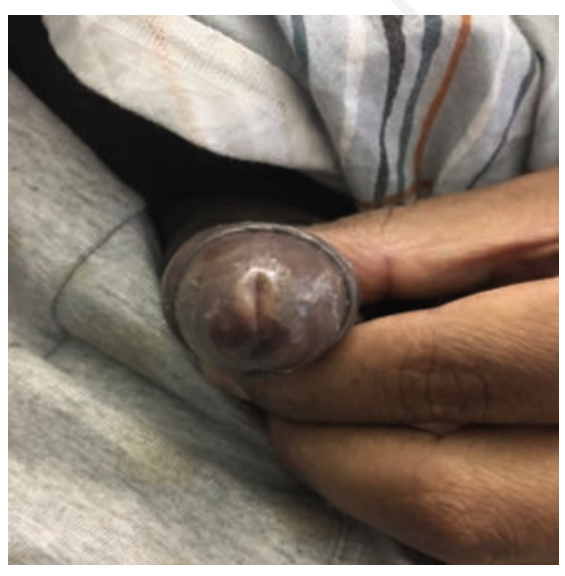

Figure 3. 16 days after stopping foscarnet the lesions had almost completely resolved $(10 / 20)$. along with him being uncircumcised, which placed him at greater risk. Lesions have also been reported, though less frequently, in women and circumcised men. A single case has been reported in a circumcised child. The fewer cases reported in women can be explained by the fact that between 19901996, foscarnet was mainly used in AIDS affected homosexual males. ${ }^{14-17}$ Most cases of ulceration have been reported to occur early on in treatment, when high dose regimens are used. ${ }^{2}$ Our patient is an exception given the sudden onset of the ulcers three weeks after initiation of foscarnet therapy.

Foscarnet-induced genital ulcers are best managed by stopping the infusion of the drug. Preventative measures are important, paying careful attention to personal hygiene with delay retraction of the prepuce and saline washing after each micturition. Washing genitals after each urination should be encouraged and probably helps reduce occurence. ${ }^{3,5,11}$

\section{Conclusions}

Genital lesions are a relatively common inpatient problem for uncircumcised males as a result of foscarnet therapy. A careful physical examination along with a detailed history and the exclusion of an infectious disease processes should in most cases be enough to make a confident diagnosis. A biopsy should be unnecessary in most cases. It is important that physicians are not only able to correctly diagnose this potential complication of foscarnet, but most importantly, that they know how to prevent it. Preventing it could save the patient from considerable morbidity which could necessitate stopping the drug. All uncircumcised patients receiving IV foscarnet therapy should be advised to wash their prepuce following each micturition.

\section{References}

1. Gerard L, Salmon-Ceron D. Pharmacology and clinical use of foscarnet. Int $\mathrm{J}$ Antimicrob Agents 1995;5:209-17.

2. Torres T. Foscarnet-induced penile ulceration. Acta Dermatovenerol Alp Pannonica Adriat 2011;20:39-40.

3. Jayaweera DT. Minimising the dosagelimiting toxicities of foscarnet induction therapy. Drug Saf 1997;16:258-66.

4. Jacobson MA. Review of the toxicities of foscarnet. J Acquir Immune Defic Syndr 1992;5:S11-7.

5. Moyle G, Barton S, Gazzard BG. Penile ulceration with foscarnet therapy. Aids 1993;7:140-1.

6. [No authors listed]. Penile ulcerations with foscarnet. Lancet 1990;335:547-8.

7. [No authors listed]. Risk of ulceration from foscarnet. Nurs Stand 1990;4:16.

8. Luelmo J. [Genital ulcers from foscarnet: three cases]. Med Clin (Barc) 1996; 107:318

9. Papini M, Bruni PL. [A case for diagnosis: penile ulcer induced by foscarnet]. Ann Dermatol Venereol 1996;123:67980 .

10. Gross AS, Dretler RH. Foscarnetinduced penile ulcer in an uncircumcised patient with AIDS. Clin Infect Dis 1993;17:1076-7.

11. Schiff TA, Bodian AB, Buchness MR. Foscarnet-induced penile ulceration. Int J Dermatol 1993;32:526-7.

12. Miguelez M. [Foscarnet and genital lesions]. Med Clin (Barc) 1993;100:717-8.

13. Evans LM, Grossman ME. Foscarnetinduced penile ulcer. J Am Acad Dermatol 1992;27:124-6.

14. Aguirrebengoa K. [Foscarnet related vulvar ulcers. Description of 2 AIDS patients]. Enferm Infecc Microbiol Clin 1995;13:315-6.

15. Caumes E. Foscarnet-induced vulvar erosion. J Am Acad Dermatol 1993;28:799. 